\title{
Using Quantitative and Qualitative Methods to Pretest the Publication Take Charge of Your Diabetes: A Guide for Care
}

\author{
LYNDA A. ANDERSON.PhD: DAWN SATTERFIELD, C-ANP.RN. MSN.CDE; ROBERT GERMAN. \\ MPH: ROBERT M. ANDERSON. EID
}

Quantitative and qualitative methods used to pretest the guidebook Take Charge of Your Diabetes: A Guide for Care are presented in this paper:

Questionnaires were used as the quantitative method (completed by 59 diabetes educators and 301 people with diabetes) and focus groups were used as the qualitative method (3 groups composed of 22 black men and women with diabetes) to examine the relerance, purpose. content. and presentation of the Guide. Findings from between-methods triangulation supported the relevance, clarity of messages, identification of groups that would be most likely to benefit, readability, understandability; and credibility of the Guide. Specific areas that needed modification were identified. Each evaluation method provided unique data; for example quantifiable data on intention to change behavior was provided fiom one method and a recommendation that diversity be maintained was provided from the other method. The relative strengths and limitations of combining quantitative and qualitative approaches are described.
The importance of incorporating the needs and perspectives of people with diabetes into diabetes education programs has long been recognized.-2 Based on this principle, pretesting of diabetes education materials before widespread dissemination is recommended. Both quantitative and. less often. qualitative ${ }^{3}$ approaches have been applied. The quantitative strategy is consistent with the major goal of pretesting. which is to collect measurable data on the extent to which the purpose and objectives of the materials are understood. ${ }^{+}$But in other areas of diabetes research, ${ }^{5-7}$ qualitative methods such as focus groups are being used to obtain more personalized feedback on the beliefs, attitudes, and behaviors of participants. which may be missed through quantitative methods." Although combining quantitative and qualitative strategies maty strengthen pretesting, there is a dearth of studies linking these approaches in the diabetes education literature.

In this paper the experience of using quantitative and qualitative methods to pretest the guidebook Take Charge of Your Diabe'es: A Guide for Care (English version, herein after termed the Guide')" is described. Quantitative feedback first was obtained from diabetes educators and people with diabetes through self-administered questionnaires on the perceived usefulness and appropriateness of the Guide. Qualitative feedback then was obtained from people with diabetes on their perceptions of the Guide and areas that needed modification. The purpose of examining combined results from quantitative and qualitative approaches was to identify specific areas in the Guide that need to be modified, and to share the findings about the relative usefulness and limitations of combining these approaches.

\section{Background: Development of the Guide}

The mission of the Division of Diabetes Translation of the Centers for Disease Control and Prevention (CDC) is to

From the Division of Adult and Community Health (Dr Anderson), the Division of Diabetes Translation (Ms Satterfield), and the Division of Cancer Prevention and Control (Mr German). Centers for Disease Control and Prevention. Allanta. Georgia and the University of Michigan Diabetes Research and Training Center, University of Michigan, Ann Arbor, Micligan ( Dr Anderson).

Correspondence to Lynda Anderson. PhD. Centers for Disease Control and Prevention. Nattional Center for Chronic Disease Prevention and Health Promotion (K-3()), 4770 Buford Hwy. NE, Atlanta GA 30341-3724.

Reprint requests to The Diaberes Educaror. 367 West Chicago Avenue, Chicago IL 60610. 
reduce the burden of diabetes by ensuring that diabetes research findings are translated into improved clinical and public health practice. ${ }^{\text {" }}$ Print materials play a critical role in comprehensive diabetes education programs ${ }^{11-12}$; this form of communication can reinforce instructions and lend itself to more detailed message delivery than oral forms of communication used alone. ${ }^{13}$ One example of print materials developed by the Division to promote quality diabetes care is the 1991 guidebook The Prevention and Treatment of Complications of Diabetes: A Guide for Primary Care Practitioners. ${ }^{1+}$ ${ }^{15}$ More recently, the Division developed a companion guide, Take Charge of Your Diabetes: A Guide for Care". The purpose of the Guide is to promote the involvement of people with diabetes in their care and to prevent complications by providing recommendations about diabetes self-care. The Guide is designed to complement direct instruction by practitioners.

The Guide has four goals: (1) to deliver messages about prevention and self-care activities to people with diabetes, (2) to encourage individuals to ask members of the diabetes care team about diabetes-specific management. (3) to facilitate behaviors that should occur at regular intervals (eg. obtaining annual dilated eye examinations), and (4) to promote documentation and record-keeping of other diabetesrelated activities (eg, sick-day care). The text and illustrations in the Guide promote self-care by increasing knowledge and improving personal record-keeping. Flowsheets are incorporated that address such topics as concerns to be discussed and tests to be completed at upcoming clinic visits; readers are reminded throughout the text to record this information on the flowsheets. The flowsheets are distinguished by a yellow background and are designed so that readers can record diabetes-specific management notes for up to 6 years.

The Guicle was pretested as part of the formative evaluation process. Pretesting helps establish the personal and professional relevance of materials, identify strong and weak points in the text. fine-tune the wording and visual images. and determine the cultural appropriateness of a document before widespread dissemination. ${ }^{13.16}$ Thus, pretesting the Guide offered CDC a chance to improve the document before additional resources were committed to produce a finished product, in this case another edition.

The framework for developing and evaluating the Guide was the health communication "wheel" that the CDC previously had created as part of a broader movement to integrate health communications into prevention programs. ${ }^{17}$ The 10-step process from the health communication wheel began with reviewing information about current gaps in diabetes education materials, determining communication objectives, identifying and segmenting the intended audience. selecting communication channels, and pretesting for the relevance and readability of materials. ${ }^{17}$

Initial review by members of the American Association of Diabetes Educators (AADE) revealed a need to lower the reading level of the Guide, ${ }^{18.19}$ which then was adjusted to a 6th-grade standard. Other characteristics of the Guide that affected how easy it was to read and understand were graphics, print style, organization of ideas, amount of preexisting knowledge required by the reader. and anount of information presented. Thus, information was reinforced through illustrations, flowsheets were developed as described earlier. A preliminary version of the Guide then was developed and subjected to the additional pretesting described in this report.

\section{Methods}

Study Design A quantitative approach was determined to be necessary for collecting data that would measure the extent to which the Guicle was viewed as useful to practice, appropriate to specific audiences, capable of enhancing knowledge about diabetes care and health care behaviors, and able to influence an individual's intentions to alter specific diabetes care behaviors. Early in the research process. however, we became aware that quantitative information alone would not provide sufficient guidance for improving the Guide. Thus, a decision was made to collect qualitative data that would enrich the pretesting findings. Focus group research was selected because it permits in-depth probing for information, which is not possible using a questionnaire. The focus group initiative was to obtain feedback on the understandability and cultural appropriateness of the Guide from a minority group perspective. ${ }^{211}$ Black men and women were chosen als the focus because this minority group was underrepresented in the written survey. The informal homogeneous groups and open-ended nature of the questions were thought to encourage participants to share their thoughts and feelings."

\section{Quantitative Data: Self-Administered Questionnaires}

Procedures In 1992 the Division of Diabetes Translation entered a collaborative agreement with the AADE to pretest the Guide among diabetes educators and their clients. A nonprobability sample of diabetes educators was obtained through an announcement in the $A A D E$ New'sletter. As part of this process. respondents were asked to indicate the number of copies of the Guide (up to 10) and corresponding questionnaires they needed to distribute. Then each respondent was sent a diabetes educator questionnaire along with a packet of copies of the Guide and client questionnaires. Explicit instructions were provided for selecting eligible participants from the practice. In brief. respondents were asked to select a start date randomly and then have an office staff member randomly select a number between 1 and 10 . For example, if the respondent selected the following Wednesday and the staff person selected the number 3. the third client seen on that Wednesday was invited to participate in the study. After identifying the first candidate, respondents were requested to ask each subsequent client to participate in the study until all the questionnaires and copies of the Guide were distributed.

Study participants were given a copy of the Guide to review at home and were asked to complete and return the questionnaire within 3 to 4 weeks. The research team was blinded to the identity of participants; all information was collected by participant numbers. Educators who consented to participate in this study followed the informed consent procedures stipulated by their institutions for recruiting research subjects. Only data for participants over the age of 18 years are included in this report.

Measures The questionnaire for diabetes educators requested demographic and background information about 
Table 1. Key Issues by Information Source

Issue
Relevance
Teaching
Resource for different groups
Appropriateness
Purpose
Most important message
Prevention/self-care
Involvement in care
Behavior change
Promote record-keeping
Content
Readability
Topics
Suggested revisions
Presentation/Style
Format
Illustrations
Suggested revisions
$\checkmark$ Closed-ended question(s).
- Open-ended question(s).

Self-Administered Questionnaire
Diabetes Educators $\quad$ People With Diabetes

$\checkmark$

$\sqrt{2+2}$

Promote record-keeping

Content

Readability

Topics

Suggested revisions

Presentation/Style

llustrations

$\checkmark$ Closed-ended question(s)

- Open-ended question(s) their practices. In addition, evaluation was sought on various aspects of the Guide through closed- and open-ended questions (Table 1. column one). The questionnaire contained four items that addressed the educator's perceptions of the utility of the Guide in terms of teaching and seven items regarding their perceptions about its use as a resource for specific groups of individuals with diabetes. Two items were included about the ability of the Guide to facilitate self-care management skills and promote involvement in diabetes care, and one item each about perceived readability and coverage of important topics. A comment section also was provided for making suggestions about revisions.

The questionnaire for people with diabetes was used to collect information on demographics, personal history of diabetes, and current diabetes care practices. As with the educators, both questionnaires contained closed- and openended questions (Table 1. column two). One item each was included on the degree to which the Guide provided a prevention message and promoted personal involvement in care: one item also addressed their perception of the readability of the document. Nine items were included about the degree to which the Guide influenced the reader's intentions to alter personal behavior. In the final section were three open-ended questions about the Guide: What was the most important message the respondents obtained from it? What did they like about it? How could it be improved? Copies of the questionnaires are available from the authors upon request.

Sample Fifty-nine healthcare professionals completed and returned a questionnaire $60 \%$ of those who inquired about the survey). This convenience sample was composed largely
(94\%) of certified diabetes educators: nurses comprised $78 \%$ of the sample, with $40 \%$ having a master's degree or higher. The mean age of respondents was 46.5 years $(S D=9.3$ ); the mean number of years they had provided patient education was 13.3 years $(\mathrm{SD}=8.3)$ and diabetes education was 9.8 years $(S D=7.3)$.

The questionnaire for people with diabetes was completed and returned by 301 respondents, representing a mean of 5.1 questionnaires per educator $(S D=2.3)$. Approximately $63 \%$ of the respondents were women. The mean age of the simple was 54.4 years $(S D=15.6)$, the majority of respondents $(86 \%)$ were white, $46 \%$ reported an annual income of $\$ 20000$ or greater, and $43 \%$ had a high school education or greater. Forty-six percent of respondents reported that they had non-insulin-dependent diabetes mellitus (NIDDM). $30 \%$ had insulin-dependent diabetes mellitus (IDDM), and 20\% were uncertain as to the type. This information was missing for $4 \%$. Of those who reported taking diabetes medications ( $85 \%$ of the sample), $43 \%$ took oral medications and $57 \%$ were treated with insulin. All participants were currently receiving diabetes education, and $60 \%$ indicated that they had attended a formal diabetes education program in the past.

Qualitative Data: Focus Groups Procedures In 1994 the Division of Diabetes Translation contracted with the Health Promotion Council (HPC) of Southeastern Pennsylvania to conduct focus groups with black men and women to pretest the Guide. Recruitment was designed to yield three groups of people defined by varying lengths of experience with the disease and different levels of accessibility to diabetes education classes. HPC recruited diabetes educaltors from seven publicly funded municipal health centers in Philadel- 
phia to solicit volunteers for the focus groups. The Guide was distributed to the participants" residences several weeks prior to the focus group meetings. HPC held focus groups at two public health centers; sessions lasted approximately $1 \frac{1}{2}$ hours. A $\$ 50$ honorarium was provided to each participant. Each session had two moderators who were black, one served as the principal moderator and the other as the moderator's assistant and recorder.

Measures The focus group used a discussion guide containing the following core areas: relevance, purpose, content, and presentation of the Guide. The discussion guide is available from the authors upon request. A series of predefined questions were used to elicit discussion within each area (Table 1, column 3). Groups were audiotaped with the participants consent.

Sample There were 22 participants in the three focus groups, 15 black women and 7 black men. Participants ranged in age from 35 to 81 years. All participants were receiving health care at a publicly funded health center or medical clinic that accepted Medicaid patients. Of the 19 participants for whom employment status was available, 12 were retired or unemployed. Of the 17 persons who indicated their educational attainment, one third had completed less than high school, another third had a high school education, and the remaining third had 1 or 2 years of college.

Data Analysis Standard statistical techniques were used to analyze the quantitative data. Response options were collapsed from five to three categories for the closed-ended questions (ie, Strongly Agree and Agree were combined into one category, No Opinion was retained as the middle category, and Strongly Disagree and Disagree were combined into the third category). In terms of open-ended responses, the first two authors (LAA and DS) independently grouped these responses into categories. All reported responses that are presented were categorized by consensus of the authors.

The techniques used to analyze the qualitative data ${ }^{8}$ were suggested in the literature. The moderator and assistant moderator listened to audiotaped transcripts and read meeting notes; bilsed on these two data sources they identified major themes and findings from the participants' responses and described them in a written report. ${ }^{21}$

Between-methods triangulation was used to link and compare data from the cuantitative and qualitative approaches. ${ }^{21-2.3}$ Between-methods triangulation refers to the use of dissimilar methods to generate and compare data about the same phenomenon. ${ }^{21}$ In this study frequency distributions and open-ended responses from questionnaires were contrasted with the themes obtained from the content analysis of the focus groups. The areas of overlap between the approaches are shown in Table 1.

\section{Results}

The results are summarized in three sections: findings from the between-methods triangulation, quantitative findings in which no comparable qualitative data were available, and qualitative findings in which there were no comparable quantitative data.
Between-Methods Triangulation Comparative data from the questionnaires and focus groups are shown in Table 2. Both methods revealed that the messages of the Guide were understood by the intended audience (ie, people with diabetes indicated that the Guide had a self-care/prevention orientation, increased knowledge, and encouraged active involvement in their diabetes care). In terms of suggested revisions, a number of the diabetes educators highlighted the need to identify the intended audiences and state the objectives clearly in the introductory section. Participants in the focus groups underscored the importance of having a message that was suitable for all ethnic groups and recommended that the Guide continue to reflect cultural diversity but not be tailored to a specific ethnic population.

Both methods confirmed that the Guide was understandable and credible (Table 2). All three groups of respondents (the two groups who answered the questionnaires and the focus groups) indicated that more information was needed in the Guide on nutrition and physical activity. Participants in the focus groups suggested that supplementary materials should be cited so that information not available in the Guide could be easily located. All respondent groups agreed that the flowsheets should be made portable. Diabetes educators also suggested that the flowsheets include sheets for monitoring lifestyle behaviors such as physical activity.

Quantitative Data All of the educators agreed that the Guide had value as a home reference for individuals. $98 \%$ indicated that it was useful as a teaching tool, $98 \%$ indicated that it provided recommendations consistent with what is currently taught, and $91 \%$ indicated that it was accurate [data not shown in tables]. The educators viewed the Guide as most useful for people with NIDDM on oral medications. then for people with NIDDM with diet/physical activity prescriptions, and then for people with NIDDM using insulin. Although over $85 \%$ of responding educators indicated the Guide would be useful for older adults, less then half believed it would be useful for women with IDDM planning to become pregnant or for parents of children with diabetes.

Respondents with diabetes were very likely $(95 \%)$ to agree that they would recommend the Guicle to family and friends. Respondents' reports of current behavioral patterns as well as their intentions to alter each behavior after reading the Guide are shown in the Figure. For example. although $45 \%$ of respondents indicated that they had their urine checked every year for albumin or protein. more than $52 \%$ reported that they intend to request this test as a result of reading the Guide. More than $30 \%$ of respondents indicated that they did not know they should get dilated eye exams. check their feet daily, and wear identification but reported they planned to do these things after reading the Guide. Over $90 \%$ of respondents indicated that they already knew about wearing shoes that fit. All reported that they knew or intended to follow illness care procedures.

Qualitative Data Respondents indicated that the Guicle was appropriate for many groups but would be especially useful for people who were either newly diagnosed or who had not yet attended diabetes education classes [datta not shown in tables]. Participants noted that the spiral binding, large print, and tabs separating sections enhanced the appeal 
Table 2. Major Findings From Between-Methods Triangulation

\section{Issue \\ Purpose}

Message

Suggested revisions

Content

Readability

Suggested revisions

Presentation/Style

Suggested revisions
Quantitative Method: Questionnaires

- Ninety-five percent of people with diabetes agreed that the Guide he!ped them understand how to prevent problems.

- Eight-four percent of people with diabetes agreed that they would discuss the Guide' with their provider.

- Comments from people with diabetes revealed that the Guide provided new information, taught new skills, promoted an active role. and entanced coping skills.

- Twenty percent of diabetes educators made recommendations suggesting that the introductory section be expanded to identify the intended audience and objectives of the Guide.

- Eighty-eight percent of the diabetes educators indicated that the Guide was easy to read and at an appropriate reading level.

- Twenty comments from educators suggested expanding the amount of information about nutrition, meal planning, and physical activity.

- Forty comments from people with diabeles suggested including more information on diet and exercise
Qualitative Method: Focus Groups

- Participants indicated that the Guide had the following attributes: encouraged them 10 ask questions and work with providers as part of a toam. made it casier for them to plan diabetes-related activities, and gave them information about how to manilge diabetes.

- Participants indicated that the Guride would serve ats a useful resource in the future.

- Participants indicated that the Guide should continue lo show diversity and not target a single ethnic group.

- Participants indicated that the Guide wats easy to reald and understand, fairly comprehensive. and credible.

- Participants indicated that the Guide needed to include more information on diet and exercise.

- Even though the Guide could not iddress everything. respondents suggested that more information should be given on diet and exercise.

- Participants suggested that other sources of information should he provided about where to go for more information on topics not presented.

- Participants suggested that the yellow flow sheets should be removalole.

- Participants suggested that the Guide should be designed so people can easily identify the different parts. of the Guide. Although focus group participants indicated that the Guide made it easier for people to plan and keep records of diabetes-related activities, they cited numerous difficulties with record-keeping. including not being accustomed to keeping records, having to expend too much effort to keep the records either daily or when they were sick, and their beliefs that doctors do not do all the tests anyway. In brief, participants indicated that the Guide was easy to use but that record-keeping would still be difficult even with the flowsheets. Participants also pointed out a lack of clarity in several of the formatting features, including the icon to remind people to write things down and the yellow flowsheets in the back of the book.

\section{Discussion}

Methods chosen for pretesting materials should suit the problem being investigated. In this study, questionnaires and focus groups were employed to generate and collect data about key issues important in pretesting the guidebook Take Charge of Your Diabetes: A Guide for Care." and betweenmethod triangulation was used to compare and contrast the findings. The aim of using these two approaches, which were thought to be complementary, was to examine the relevance, purpose, content, and presentation of the Guide. Quantitative data was sought to document the extent to which specific beliefs and opinions were held, and qualitative information was sought to gather viewpoints from a specific minority group (blacks). The use of multiple strategies helped to validate study findings and contribute to convergence in suggested areas for revision. ${ }^{21.23}$ For example, both approaches offered supportive evidence for the relevance. clarity of messages, identification of groups most likely to benefit, realdability, understandability, and credibility of the Guide, and both indicated that specific areas needed modilication. An additional benefit was that each method potentially could uncover unique perspectives nol revealed by a single method, which was found to be the case. Each approilch provided unique data; quantifiable data on intention $(0)$ change behavior was uncovered by one method and a recommendation that diversity be maintained in the Guicle was revealed by the other method.

Several problems are inherent in using multiple rescarch methods. ${ }^{21.24}$ The unit of analysis is likely to difler between methods; in survey research individuals are the unit of 


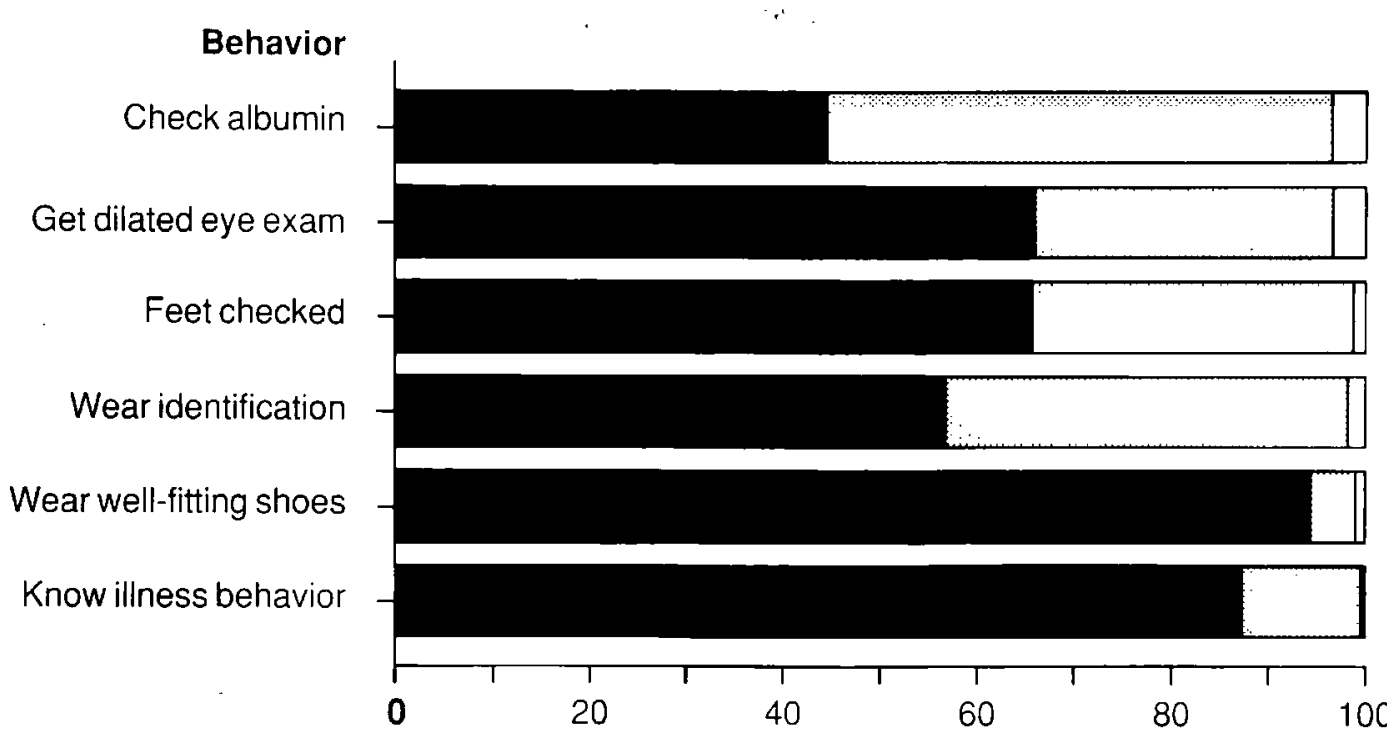

Intentions

it

Already doing $\square$ Intend to start doing $\square$ Do not know

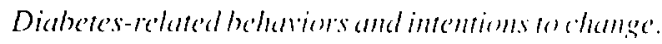

\section{Table 3. Modifications made to Take Charge of Kour Diabetes: A Guide for Care}

Purpose

Expand the prefice to include statements about intended atudience objectives of the Guide. and use of Guide to supplement education programs.

Restate the purpose of the Guide in the introduction and clarily that the scope is restricted to prevention of complications. Content

Add a brief overview of diabetes to the introduction and explain the value of balancing nutrition. physical activity, and medications in achieving glucosecontrol.

Add more discussion of stress and support issues in the section on leelings about diabetes.

Write an introduction to the record-keeping section addressing some of the barriers to use and emphasizing the benefits of record-keeping.

Presentation/Style

Reduce the number of llowsheets to those that assist in monitoring lests and minimize the grid appearance.

Add glossary of terms.

Remove hand icon for recording inlormation.

Provide captions for illustrattions that describe the positive actions the person is performing.

Add new simple line drawings depicting positive activities.

Continue to use illustrations that depict people from different cultures in a respectful way.

analysis, whereas in focus group research the group is the unit of analysis. As a result there is no cortainty that the data are comparable across melhods. Costs also are greater when two methods are used than when either method is used alone. In addition, the complexity of combining. analyzing, and interpreting large amounts of dissimilar data can be overwhelming.

Other limitations also should be noted. Convenience samples, which were relied on in this study, frequently have limited generalizability. Convenience samples maly be bialsed because exposure to the materials cannot be controlled and a certain level of reading and writing skills is required to com- plete the questionnaire and read the Guide. In addition, the findings from the focus groups cannot be generalized to the larger population of blacks because the groups were from a specific geographic area and the number of participants was quite small.

A final summary of the revisions that are being made in the Guide batsed on the lessons learned from these pretesting activities is presented in Table 3. The CDC is incorporating the majority of recommendations from both the people with diabetes and the diabetes educators. The revisions addressing the recommended expansion of information on nutrition and physical activity will include a new introductory section that 
emphasizes the importance of these activities in achieving good glucose control. Additionally, readers will be directed to other sources to obtain specific guidance.

This study offers an example of using between-methods triangulation to link quantitative and qualitative methods. There are a variety of forms of triangulation (eg, data, investigator, and methods), ${ }^{21}$ all of which can be applied to a number of research issues including hypothesis generation, program evaluation, and development of quantitative measures. A number of studies ${ }^{22-25}$ in health education and nursing research have linked quantitative and qualitative research; this study is one of the first to do so in the diabetes education literature. As diabetes educators continue to champion the necessity of pretesting their materials in a rigorous fashion, it is hoped that this process can be enhanced by partnering quantitative and qualitative methods to gather data and combining these methods through triangulation. The authors hope that this paper illustrates the strengths and limitations of combining qualitative and quantitative methods in pretesting diabetes education materials.

The authors acknowledge the contributions of the following individuals in facilitating the survey with the AADE membership: Linda Haas, Betty Brackenridge, Julie Meyer, and Jean Betschart. The authors also thank Janet Ohene-Frempong and Delores Merrick for their leadership in the focus group research and recognize the valuable contribution of Patricia Mitchell throughout the development process of the Guide.

\section{References}

1. Funnell MM, Haas LB. National standards for diabetes self-management education programs. Diabetes Care 1995:18:100-16.

2. Duchin SP, Brown SA. Patients should participate in designing diabetes educational content. Patient Educ Counsel 1990;16:255-67.

3. Hosey GM, Freeman WL, Stracqualursi F. Gohdes D. Designing and evaluating diabetes education material for American Indians. Diabetes Educ 1990;16(5):407-14.

4. Steckler A, Eng E, Goodman RM. Integrating qualitative and quantitative evaluation methods. Hygiene 1991/2;10:16-20.

5. Quatromoni PA, Mibbauer M, Posner BM, et al. Use of focus groups to explore nutrition practices and health beliefs of urban Caribbean Latinos with diabetes. Diabetes Care 1994:17:869-73.

6. Anderson RM, Barr PA, Edward GJ, el al. Using focus groups to identify psychosocial issues facing urban African Americans with diabetes. Diabetes Educ 1996:22(1):28-33.

7. Satterfield DW, Edgar TM, Erlich $A B$, et al. Assessing community perceptions of diabetes using a qualitative education tool. Diabetes 1996;45(2):44A.

8. Kingry MJ. Tiddje LB, Friedman LL. Focus groups: a research technique for nursing. Nurs Res 1990;39:124-25.

9. Centers for Disease Control and Prevention. Take charge of your diabetes: a guide for care. Atlanta, Ga: US Department of Health and Human
Services, Public Heialth Service, Centers for Disease Control, Division of Diabetes Translation, 1992.

10. National Diabetes Advisory Board. The national long-range plan to combat diabetes. Washington. DC: National Institutes of Health, 1988; NIH publication no. 88-1587.

11. Funnell MM, Donnelly MB, Anderson RM, Johnson PD, Oh MS. Perceived effectiveness, cost, and availability of patient education methods and materials. Diabetes Educ 1992; 18(2):139-45.

12. Yasenchak PA, Bridle MJ. A low-literacy skin care manual for spinal cord injury patients. Patient Educ Couns 1993:22:1-5.

13. Bernier MJ, Yasko J. Designing and evaluating printed education materials: model and instrument development. Patient Educ Couns 1991: 18:253-63.

14. Centers for Disease Control and Prevention. Prevention and treatment of complications of diabetes: a guide for primary care practilioners. Atlanta, Ga: US Department of Health and Human Services. Public Health Service, Division of Diabetes Translation. Centers for Disease Control, 1991.

15. Satterfield D. Lyons A. Development of a consensus publication by the Centers for Disease Control (CDC): the prevention and trealment of complications of diabetes - a guide for primary care practitioners. Diabetes Educ 1992; 18(6):473-75.

16. Alcohol, Drug Abuse, and Mental Health Administration. Pretesting is essential; you can choose from various methods. Washington, DC: Public Health Service. Office for Substance Abuse Prevention. US Department of Health and Human Services, 1992; ADAMHA publication no. MS428.

17. Roper WL. Health communication takes on new dimensions at CDC. Public Health Rep 1993;108:179-83.

18. Hilltleman D. Readability, readability formulas, and cloze: selecting instructional materials. J Reading 1987;22:117-22.

19. Pastore PO, Berg BK. The evaluation of patient education materials: focus on readability. Patient Educ Counsel 1987;9:216-19.

20. Health Promotion Council of Southeastern Pennsylvania Health Literacy Project. A focus group evaluation of Take Charge of Your Diabetes: A Guide for Care. Philadelphia, Pa: submitted to CDC. Division of Diabetes Translation, April, 1994; project no. 93-231.

21. Duffy ME. Methodological triangulation: a vehicle for merging quantitative and qualitative research methods. IMAGE: J Nurs Scholar 1987; 19:130-33.

22. Breitmayer BJ. Ayres L, Knafl KA. Triangulation in qualitative research: evaluation of completeness and contirmation purposes. IMAGE: J Nurs Scholar 1993:25:237-43.

23. Banik BJ. Applying triangulation in nursing research. Applied Nurs Res 1993;6:47-52.

24. Buchanan DR. An uneasy alliance: combining qualitative and quantitative research methods. Health Educ Q 1992:19:117-35.

25. Steckler A, McLeroy KR. Goodman RM. Bird ST. McCormick L. Toward integrating qualitative and quantitative methods: an introduction. Health Educ Q 1992;19:1-8. 